\title{
BMJ Open Virtual reality for relatives of ICU patients to improve psychological sequelae: study protocol for a multicentre, randomised controlled trial
}

\author{
Johan H Vlake (D) , ${ }^{1,2}$ Jasper van Bommel, ${ }^{2}$ Evert-Jan Wils, ${ }^{1}$ Tim Korevaar, $^{3}$ \\ Merel E Hellemons, ${ }^{4}$ Eva Klijn, ${ }^{2}$ Anna FC Schut, ${ }^{5}$ Joost AM Labout, ${ }^{6}$ \\ Marten P Van Bavel, ${ }^{2}$ Margo MC van Mol (D) ,2 Diederik Gommers, ${ }^{2}$ \\ Michel E van Genderen (D) ${ }^{2}$
}

To cite: Vlake $\mathrm{JH}$, van Bommel J, Wils E-J, et al. Virtual reality for relatives of ICU patients to improve psychological sequelae: study protocol for a multicentre, randomised controlled trial. BMJ Open 2021;11:e049704. doi:10.1136/ bmjopen-2021-049704

- Prepublication history and additional supplemental material for this paper are available online. To view these files, please visit the journal online (http://dx.doi.org/10.1136/ bmjopen-2021-049704).

Received 30 January 2021 Accepted 14 September 2021

Check for updates

(c) Author(s) (or their employer(s)) 2021. Re-use permitted under CC BY-NC. No commercial re-use. See rights and permissions. Published by BMJ.

For numbered affiliations see end of article.

Correspondence to

Dr Michel E van Genderen;

m.vangenderen@erasmusmc.nl

\section{ABSTRACT}

Introduction Intensive care unit (ICU) admission of a relative might lead to psychological distress and complicated grief (post-intensive care syndrome-family; PICS-F). Evidence suggests that increased distress during ICU stay increases risk of PICS-F, resulting in difficulty returning to their normal lives after the ICU experience. Effective interventions to improve PICS-F are currently lacking. In the present trial, we hypothesised that information provision using ICU-specific Virtual Reality for Family members/relatives (ICU-VR-F) may improve understanding of the ICU and subsequently improve psychological well-being and quality of life in relatives of patients admitted to the ICU.

Methods and analysis This multicentre, clustered randomised controlled trial will be conducted from January to December 2021 in the mixed medical-surgical ICUs of four hospitals in Rotterdam, the Netherlands. We aim to include adult relatives of 160 ICU patients with an expected ICU length of stay over 72 hours. Participants will be randomised clustered per patient in a 1:1 ratio to either the intervention or control group. Participants allocated to the intervention group will receive ICU-VR-F, an information video that can be watched in VR, while the control group will receive usual care. Initiation of ICU-VR-F will be during their hospital visit unless participants cannot visit the hospital due to COVID-19 regulations, then VR can be watched digitally at home. The primary objective is to study the effect of ICU-VR-F on psychological well-being and quality of life up to 6 months after the patients' ICU discharge. The secondary outcome is the degree of understanding of ICU treatment and ICU modalities. Ethics and dissemination The Medical Ethics Committee of the Erasmus Medical Centre, Rotterdam, the Netherlands, approved the study and local approval was obtained from each participating centre (NL73670.078.20). Our findings will be disseminated by presentation of the results at (inter)national conferences and publication in scientific, peer-reviewed journals.

Trial registration number Netherlands Trial Register ( TrialRegister.nl, NL9220).

\section{INTRODUCTION}

An intensive care unit (ICU) admission is known to be a stressful experience for both
Strengths and limitations of this study

- A randomised controlled trial examining the effect of Intensive Care Unit-specific Virtual Reality for Family members/relatives (ICU-VR-F) on psychological well-being and quality of life using an innovative and uniform modality.

- ICU-VR-F represents an easy applicable, safe and immersive modality to improve communication through better information provision regarding treatment-related and environment-related information about the ICU, enabling relatives to receive uniform and complete information.

- ICU-VR-F is an innovative method that is generalisable and makes information easily accessible and immersive.

- Blinding of patients or investigators is not possible due to the nature of the intervention.

patients and their relatives. As a result, relatives of ICU patients are at risk of developing several psychological sequelae, such as symptoms of post-traumatic stress disorder (PTSD), anxiety, depression and complicated grief in the unfortunate event of a patient dying during ICU treatment. Clinically relevant symptoms of PTSD occur in $21 \%$ of relatives of ICU patients, especially in relatives of adult patients, clinically relevant symptoms of anxiety occur in $40 \%$ and clinically relevant symptoms of depression occur in $23 \% \cdot{ }^{1-11}$ These impairments are collectively referred to as the post-intensive care syndrome-family (PICS-F). ${ }^{6} 1213$

PICS-F frequently results in loss of employment, financial burden, lifestyle interference and a profound impact on quality of life. ${ }^{14}$ These consequences often last a long time and already start during ICU stay of their kin. ${ }^{3}$ Important risk factors for the development 
of PICS-F are the unexpectedness of critical illness, the dramatic nature of the relatives' experience leading to emotional stress, the level of communication of the ICU staff and the use of medical jargon, that frequently makes it hard for the relative to understand the treatment explanation. ${ }^{1810111516}$ As such, relatives may witness invasive treatments with unfamiliar medical procedures and devices in an environment they do not understand. Therefore, communication between ICU staff and families is essential in the care process, and good communication and information provision improve the relatives' understanding of ICU treatment and satisfaction, limit lawsuits and are associated with lower prevalence of PTSD during ICU stay. ${ }^{517} 18$ As such, good information provision to relatives of ICU patients is essential in improving the relatives' comprehension of ICU procedures and ICU environment during the ICU stay.

During the COVID-19 pandemic, many hospitals worldwide disallowed visitors for all adult inpatients including all ICU patients with COVID-19 and without COVID19. Relatives of ICU patients during the COVID-19 pandemic are therefore confronted with the impracticality of visiting their relative in the ICU or receiving good communication from the ICU staff, which may result in a higher psychological burden. ${ }^{19}{ }^{20}$ In the face of mounting PICS-F-related sequelae, several interventions, such as information brochures, family conferences and educational programmes for relatives, have been tested, but did not result in a clinically meaningful improvement in psychological well-being or quality of life. ${ }^{21} 22$ The COVID-19 pandemic has resulted in the disruption of an integral aspect of care in most ICUs across the world and the importance of generalisable and on-demand information has been addressed. To date, a clinically meaningful, simple and generalisable intervention remains unavailable.

Virtual reality (VR) is a relatively new technique that allows the user to fully immerse within a virtual environment. As such, it allows relatives to experience what the patient is experiencing during ICU treatment, possibly leading to a better comprehension of an ICU stay. Information provision using VR has shown to decrease preoperative anxiety in both adult and paediatric patients, to help women and their partner to feel better prepared for caesarean delivery, to successfully deliver healthcarerelated information to adults with intellectual disabilities and to be an appropriate tool to deliver additional treatment-related information to increase patients' satisfaction. ${ }^{23-26}$ Additionally, exposure through VR appears to be an effective treatment modality for several mental health disorders, including PTSD, depression and anxiety, in a non-ICU setting. ${ }^{27-30}$ It provides an innovative modality that is generalisable and could improve the relatives' understanding of what is happening to long-stay ICU patients, without increasing staff workload. We hypothesised that offering treatment-related and environment-related information about the ICU via VR increases relatives' understanding of ICU treatment and environment and improves psychological well-being and quality of life.

\section{METHODS AND ANALYSIS}

\section{Study design and setting}

This study will be a multicentre, clustered randomised trial conducted in the mixed medical-surgical ICUs of four hospitals in Rotterdam, the Netherlands. Cooperating hospitals are: the Erasmus Medical Centre (MC) (university hospital), Franciscus Gasthuis \& Vlietland Hospital, Ikazia Hospital and Maasstad Hospital (all teaching hospitals). The Medical Ethics Committee (MEC) of the Erasmus MC approved this study (NL73670.078.20, approved 14 December 2020), and local approval was obtained from the institutional ethics review boards of each participating hospital, that is, the Franciscus Gasthuis \& Vlietland Hospital, the Ikazia Hospital and the Maasstad Hospital. The study will be conducted from January to December 2021. Participants will be followed for 6 months after patients' ICU discharge. Any modifications to the study protocol, which may impact the conduct of the study or participant safety, including changes of the study objectives, study design, study population, sample size, study procedures or significant administrative aspects, will be sent for approval to the MEC of the Erasmus MC, and local approval will be obtained from the institutional ethics review boards of each participating hospital prior to implementation. Accordingly, the health authorities will be informed in accordance with local regulations.

\section{Study participants}

We aim to include relatives, or close friends in absence of relatives, of 160 ICU patients. Relatives $\geq 18$ years of age, who are a first/second-degree relative of the ICU patient, are responsible for decision-making or sharing the same household are eligible for inclusion. Additionally, relatives should be able to understand the Dutch language to understand ICU-specific Virtual Reality for Family members/relatives (ICU-VR-F) and should in possession of smartphone, tablet or computer to watch ICU-VR-F at home. Multiple relatives per patient can participate; the primary contact person of the ICU patient will be approached first and will be invited to share the study information with other relatives who could be interested in participation. There is no maximum number of relatives per patient who can participate. In the case of multiple relatives of the same patient participating, relatives of the same patient will be clustered to the same randomisation allocation. Relatives with no formal address or relatives of patients with an expected ICU length of stay less than 72 hours will be excluded. Close friends are eligible for inclusion in the case that no relative is available. Close friends are considered close friends if they address themselves as close friends and are responsible for decision-making. Relatives of patients who die during ICU treatment will retrospectively be excluded from the main analysis. 


\section{Intervention}

Patients will be randomised to receive standard care with additional ICU-VR-F (intervention group) or standard care alone (control group).

The ICU-VR-F intervention was based on the previously described ICU-VR intervention for ICU patients and was designed by an interdisciplinary team of three intensivists, a psychologist, a former ICU patient and a VR/film director. Based on focus group meetings with this team and previous studies, the following information was included in the intervention: (1) an introduction by an intensivist and an ICU nurse to welcome the relative to the ICU and VR environment explaining daily movements at an ICU, (2) explanation of monitors and noises in an ICU room, (3) information regarding mechanical ventilation, intubation and tracheal tube suction, (4) information and necessity of central/peripheral lines and intravenous/ drips, (5) information and necessity of the treatment team and ICU workflow. ${ }^{31}{ }^{32}$ The ICU-specific VR intervention was designed with the aim of showing relevant and truthful treatment-related and ICU environmentrelated information, and was hospital specific. The point of view for the camera was the field of vision of the mock patient lying in a hospital bed. The hospital-specific ICU-VR-F from the Erasmus MC can be found https:/ / www.youtube.com/watch?v=OakhhQ32jLs, from the Franciscus Gasthuis \& Vlietland can be found https:// www.youtube.com/watch?v=cvMffRVrOE4, and from the Ikazia Hospital can be found https://www.youtube.com/ watch? $=$ cvMffRVrOE4. The uniform video script can be found in the online supplemental data.

Standard care comprises either (1) a family meeting with the treating ICU physician during the first week of ICU admission, and (2) biweekly meetings with the treating ICU physician when patients have a stay of more than 14 days according to a hospital's local protocol. Additionally, family members will always be offered a digital/ hardcopy ICU diary according to national guidelines.

\section{Study procedures}

Outcome variables will be collected at each time point (see figure 1). The primary contact person of the ICU patient will be approached by an investigator of the research team within 2 days after ICU admission and will be asked to share the study information with other relatives. In case that other relatives are interested in participation, their contact details will be shared by the primary contact person with the investigator so informed consent can be obtained. A translation of the information for participants and the informed consent form can be found in the online supplemental data. After inclusion, participants will receive a first set of questionnaires (T0) consisting of a self-composed questionnaire regarding demographics, and validated questionnaires to assess psychological well-being and quality of life. Participants are asked to fill in the first set of questionnaires retrospectively, in order to obtain a measure of participants' anxiety and depression levels and quality of life prior to
All relatives of ICU patients with an expected ICU-LOS $>72 \mathrm{~h}$ $>18$ years old

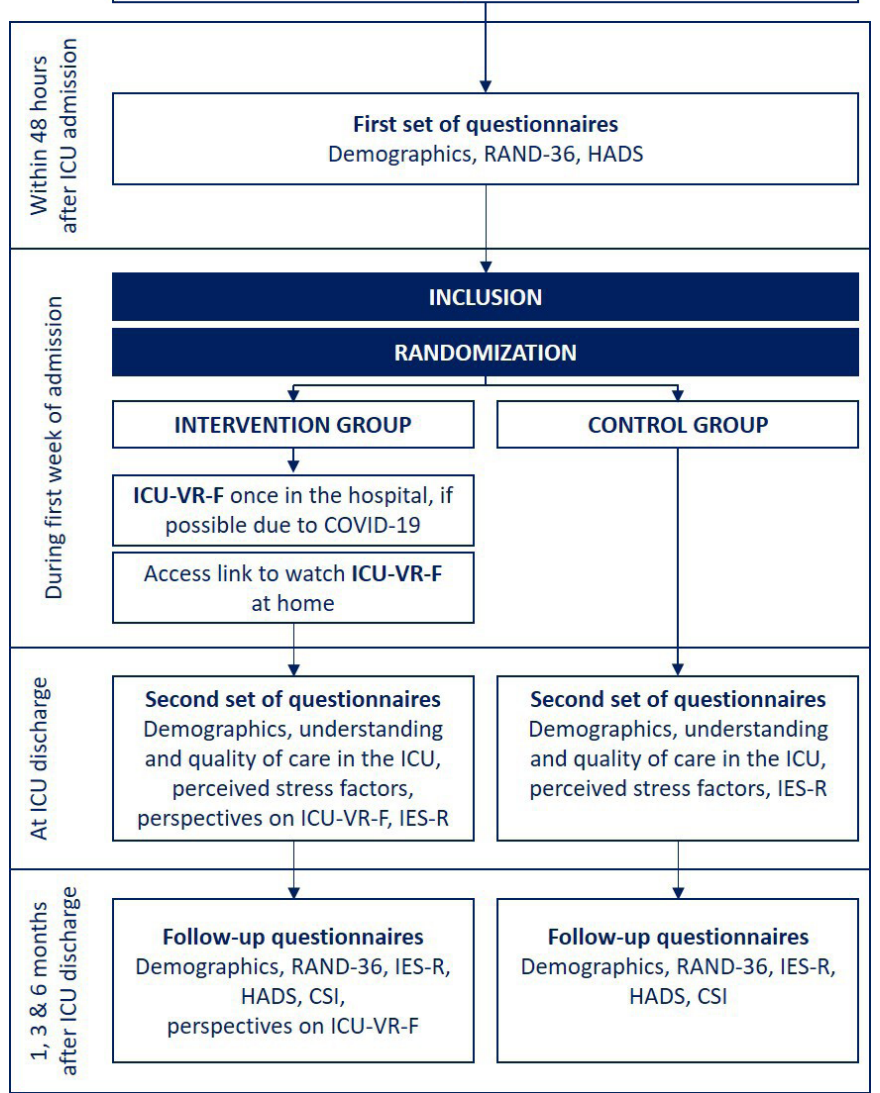

Figure 1 Flow diagram of the study. CSI, Caregivers Strain Index; HADS, Hospital Anxiety and Depression Scale; ICU, intensive care unit; ICU-LOS, ICU length of stay; ICU-VR-F, ICU-specific Virtual Reality for Family members/relatives; IES-R, Impact of Event Scale-Revised; RAND-36, Research and Development 36-item Questionnaire.

the current episode of the patient's illness leading to ICU admission. Hereafter, randomisation will be conducted.

During ICU treatment, all relatives will receive standard care, which comprises either: (1) a family meeting with the treating ICU physician during the first week of ICU admission, and (2) biweekly meetings with the treating ICU physician when patients have a stay of more than 14 days. Additionally, family members will always be offered a digital/hardcopy of an ICU diary.

After randomisation, participants in the intervention group will additionally receive ICU-VR using headmounted display VR (Oculus Go, Irvine, California, USA, CE: R-CMM-OC8-MH-A). Thereafter, they receive cardboard VR glasses and an access link to watch ICU-VR-F at home, which can also be used without the cardboard VR glasses. Participants who are not allowed to visit the hospital due to COVID-19 regulations, that is, mandatory self-quarantine, inability to visit the ICU or a limited number of visitors, will only receive ICU-VR-F using cardboard VR glasses via the access link. The number of times a participant watches ICU-VR-F will be logged. Participants will have access to the intervention during 
Table 1 Questionnaire per follow-up moment

\begin{tabular}{|c|c|c|c|}
\hline Questionnaire & $\begin{array}{l}\text { T0 } \\
\text { At ICU admission }\end{array}$ & $\begin{array}{l}\text { T1 } \\
\text { At ICU discharge }\end{array}$ & $\begin{array}{l}\text { T2/T3/T4 } \\
\text { Follow-up (1/3/6 months) }\end{array}$ \\
\hline Baseline demographics & $x$ & $\mathrm{X}$ & $x$ \\
\hline $\begin{array}{l}\text { HADS } \\
\text { (anxiety and depression) }\end{array}$ & $\begin{array}{l}\text { X } \\
\text { (retrospectively) }\end{array}$ & & $x$ \\
\hline $\begin{array}{l}\text { IES-R } \\
\text { (post-traumatic stress disorder) }\end{array}$ & & $x$ & $x$ \\
\hline $\begin{array}{l}\text { RAND-36 } \\
\text { (quality of life) }\end{array}$ & $\begin{array}{l}\text { X } \\
\text { (retrospectively) }\end{array}$ & & $x$ \\
\hline
\end{tabular}

Subset CQI-Relatives in the ICU

X

(understanding ICU procedures)

CSI

(caregiving concerns

Perceived stress factors

Perspectives on the ICU-VR-F intervention

$x$

$\mathrm{X}$

$\mathrm{X}$

CQI, Consumer Quality Index; CSI, Caregivers Strain Index; HADS, Hospital Anxiety and Depression Scale; ICU, intensive care unit; ICU-VR-F, ICU-specific Virtual Reality for Family members/relatives; IES-R, Impact of Event Scale-Revised; RAND-36, Research and Development 36-item Questionnaire.

the entire study period, including follow-up. Participants will receive a second set of questionnaires during ICU discharge of their relative to assess their understanding of ICU procedures and environment, and will receive follow-up questionnaires at 1 month, 3 months and 6 months after ICU discharge (table 1).

The study procedures of participants in the intervention group who are allowed to visit the hospital are presented in figure 2 and for those who are not allowed to visit the hospital in figure 3 .

\section{Randomisation and masking}

Randomisation will be on a 1:1 ratio, clustered based on the ICU patient (that is, if multiple relatives of one ICU patient participate, they will all be assigned to the same group), stratified for study site and the ability to visit the hospital with regard to COVID-19 regulations. Randomisation will be performed using a centralised internet-based randomisation procedure (Castor EDC, Amsterdam, the Netherlands). Due to the nature of the intervention, blinding is not possible.

\section{Outcomes and measurements}

The primary endpoint is the effect of ICU-VR-F on psychological well-being and quality of life in participants up to 6 months after ICU discharge. Psychological well-being will be expressed as the presence and severity of PTSD-related, anxiety-related and depression-related symptoms, and will be

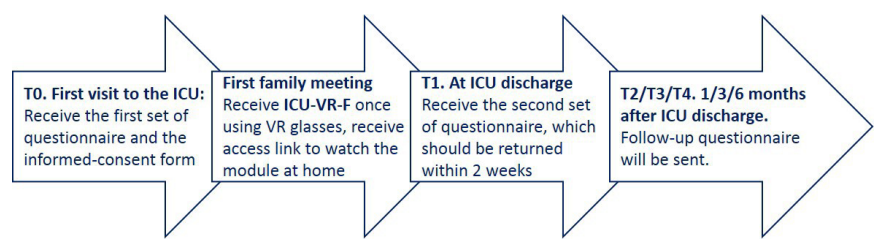

Figure 2 Overview of procedures for relatives in the intervention group who are allowed to visit the hospital. ICU, intensive care unit; ICU-VR-F, ICU-specific Virtual Reality for Family members/relatives. assessed using the Impact of Event Scale-Revised (IES-R) and Hospital Anxiety and Depression Scale (HADS). ${ }^{33}$ Quality of life will be assessed using the RAND-36. ${ }^{35} 36$ The secondary endpoint is the participants' understanding of the ICU environment and procedures, that is, devices, monitors, sounds (alarm noises) and daily work practice. Understanding of ICU procedures will be assessed using a subset of the Consumer Quality Index-Relatives in the ICU (CQI-Relatives in the ICU). ${ }^{37}$ Additional outcomes are the perceived stress factors during ICU treatment and the perspectives of participants about ICU-VR-F, assessed using the Caregivers Strain Index (CSI), a self-composed 'perceived stress factors' questionnaire, and a self-composed 'perspectives on the ICU-VR intervention' questionnaire.

The IES-R comprises 22 items, assesses subjective distress caused by a traumatic event and has been previously validated in ICU survivors. ${ }^{38} 39$ The IES-R yields a total score (ranging from 0 to 88, with higher scores indicating more severe symptoms), and subscale scores can be calculated for symptoms of intrusion, avoidance and hyperarousal. An IES-R sum score $\geq 24$ will be considered as PTSD. ${ }^{4041}$

The HADS comprises 14 items and is commonly used to determine the levels of anxiety and depression that a person is experiencing. A sum score $>8$ on either the depression (seven questions) or anxiety (seven questions) subscale will be classified as depression and anxiety, respectively. $^{3342} 43$

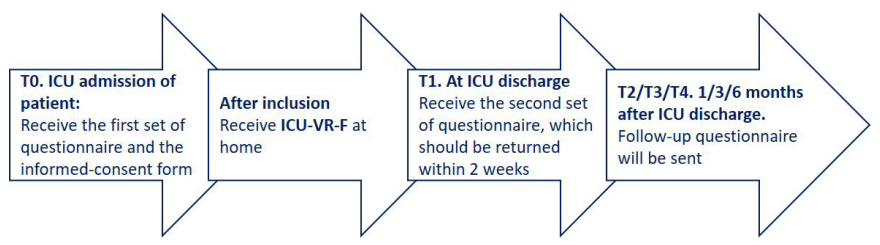

Figure 3 Overview of procedures for relatives in the intervention group who are not allowed to visit the hospital. ICU, intensive care unit; ICU-VR-F, ICU-specific Virtual Reality for Family members/relatives. 
The Research and Development 36-item Questionnaire (RAND-36) consists of 8 scaled scores, which are the weighted sums of the questions in their section. Each scale is directly transformed to a scale ranging from 0 to 100 on the assumption that each question carries an equal weight. The eight sections are vitality, physical functioning, bodily pain, general health perception, physical role functioning, emotional role functioning, social role functioning and mental health. In addition, a mental and physical component scale can be calculated, giving a perception of a person's physical and mental health. ${ }^{44}$

The CQI-Relatives in the ICU was designed by the Healthcare Institute of the Netherlands in collaboration with several hospitals to measure the perceived quality of care by relatives of ICU patients. ${ }^{37}$ The subset used in the present study was carefully tailored to the needs of the current study (online supplemental data). Therefore, unnecessary items for this study were removed and additional VR-specific questions were added. The subset consists of 38 items, distributed across 4 sections; (1) general questions, (2) questions regarding information provision and understanding of the ICU environment, (3) questions regarding care offered to relatives and (4) questions regarding the communication with the ICU staff.

The self-composed perceived stress factors questionnaire was based on existing literature regarding risk factors for the development of PICS-F, including time spent for visitation, worries about the physical, cognitive and psychological state of the patient, worries about family and familiarity with an ICU. The final questionnaire comprises 18 questions which can be answered on a Likert scale ranging from 0 (not at all) to 4 (a lot). The self-composed perspectives on the ICU-VR-F intervention questionnaire comprises 13 questions. Outcomes of these self-composed questionnaires will be used to determine different aspects of information that relatives were missing or were in need of in the current ICU-VR-F intervention. These data will be used to further improve the VR intervention and its content so it will better meet the needs of relatives. Translations of the self-composed questionnaires can be found in the online supplemental data.

\section{Data management}

Data will be uploaded, stored and maintained on the electronic data capture (EDC) system of Castor (Castor EDC (www.castoredc.com), Amsterdam, the Netherlands). The study team will be responsible for all data entry and quality control activities. The data will be checked by at least two persons from the study team and will be stored for at least 15 years on either the Castor EDC server or as a hardcopy in the ICUs of the participating hospitals. Questionnaires will be sent digitally using Castor EDC or hardcopy via postal mail whenever requested.

To maintain anonymity, data will be coded with a number and this number will be the only reference to identification. The principal investigator will be the only one in possession of the translation key, making it impossible to link data to the participant.

\section{Sample size calculation}

To the best of our knowledge, this study will be the first of its kind for which no previous conducted studies can be used to define the expected effect estimate. Due to expected non-normality of PTSD, depression and anxiety scores at 6 months after ICU discharge, this calculation could represent an overestimation of the effect estimate. Based on our clinical experience, and experience with a pilot study studying the effects of ICU-VR on ventilated ICU patients for which we found Cohen's $d$ effect size of 0.77 , we expect that a clinically meaningful Cohen's $d$ effect size of 0.55 could be expected in relatives. ${ }^{32}$ When taking this into account, using a two-sided alpha of 0.05 , and a power of 0.80 , assuming an expected loss to follow-up of $20 \%$, we aim to include relatives of $160 \mathrm{ICU}$ patients. We expect a needed time of 6 months based on the admission rate history of the participating hospitals.

\section{Statistical analysis}

Baseline demographics and treatment-related characteristics will be quantified using descriptive statistics. Continuous variables will be presented as mean (SD) or as median (95\% range), based on the distribution of the variable. Categorical variables will be presented as absolute number and relative frequency.

A sensitivity analysis will be performed in which missing data (completely) at random will be dealt with using both multiple imputation according to the Markov-chain Monte Carlo and the Last Observation Carried Forward Method. $^{45}{ }^{46}$ We will correct for multiple testing using the false discovery rate with a maximum of $5 \%$ false negatives. ${ }^{47}$

For the primary outcome, the effect of ICU-VR on PTSD, anxiety, depression and quality of life, we will analyse differences in the IES-R sum score (PTSD), the HADS anxiety and depression score, and the RAND-36 subscales (quality of life) between participants in the intervention and the control group at each follow-up time point (that is, 1 month, 3 months and 6 months after ICU discharge) and throughout follow-up using a mixed-effects linear regression model with a random intercept for each study site and/or participants based on model comparisons using the Akaiki information criteria. In case of multiple relatives of one ICU patient participating, these participants will be considered as clustered, and a random intercept for each cluster will be used. Between-group differences in variables of interest throughout follow-up were studies by introducing the product of timextreatment group to the model.

Differences in the proportion of participants in the intervention group and participants in the control group with clinically relevant symptoms of PTSD (IES-R sum score $\geq 22$ ), depression (HADS depression score $>8$ ) or anxiety (HADS anxiety score $>8$ ) will be analysed using a mixed-effects logistic regression model. Also, changes 
from baseline will be computed dividing the parameter value at specific time points into the baseline value expressed as percentile changes (\% of baseline). The magnitude of change among PTSD, depression, and anxiety at specific time points and differences will be tested using a mixed-effects linear regression model.

For the secondary outcome, understanding of the ICU and quality of care in the ICU, we will analyse differences between study groups per question using a mixed-effects logistic regression model. By combining the numerical values of the answers given, a sum score and subscales for the different sections can be calculated for each participant. The association between the intervention and these sum scores will be examined using mixed-effects linear regression models.

The explorative outcomes, the perceived stress factors and the perspectives of relatives on the ICU-VR-F intervention, will be described using descriptive statistics. Differences in continuous outcomes of the self-composed questionnaire regarding perceived stress factors and the sum score of the CSI will be analysed using mixed-effects linear regression models. Differences in categorical outcomes of the self-composed questionnaire regarding perceived stress factors will be analysed using mixedeffects logistic regression models.

The main analyses will be conducted per protocol. In these, all patients who have received ICU-VR-F, either both in the hospital and at home or only at home, will be compared with those who did not, and relatives of patients who have died during ICU treatment will be excluded. To determine whether there is a difference in effect between having watched ICU-VR-F the first time in the hospital and having watched the ICU-VR-F only at home, we will use dummy variables (ICU-VR-F in the hospital and at home/ICU-VR-F only at home/no ICUVR-F) instead of the randomisation variables in the mixed-effects regression models, and determine whether that dummy variable has a significant contribution to the model. We will additionally perform an analysis in which (1) patients who did not watch ICU-VR-F in the hospital will be excluded and (2) patients who watched ICU-VR in the hospital will be excluded to determine whether there is a difference in effect.

All data will be gathered using Castor EDC. Analyses will be performed using SPSS (V.27.0) and R for Statistics (R Foundation for Statistical Computing, Vienna, Austria, 2015). A $p$ value of $\leq 0.05$ will be considered statistically significant.

\section{Ethics and dissemination}

This study will be conducted in accordance with the principles of the Declaration of Helsinki (version October 2013; www.wma.net) and in accordance with the Medical Research Involving Human Subjects Act and other guidelines, regulations and acts. We received approval from the MEC of the Erasmus MC, and local approval has been obtained from the institutional ethics review boards of each participating hospital, that is, the Franciscus
Gasthuis \& Vlietland Hospital, the Ikazia Hospital and the Maasstad Hospital. If deviation from the protocol is necessary, then it will not be implemented without the prior review and approval of the MEC of the Erasmus MC and each participating hospital's institutional ethics review board. Signed informed consent will be obtained from all participants. Previous research demonstrated that (ICU-)VR is safe. ${ }^{23} 313248$ Informed consent forms will be kept in a locked cabinet in a limited-access room at the Erasmus MC. Data will be archived for 15 years. The handling of personal data complies with the Dutch law. On completion of the study, its findings will be published in peer-reviewed journals and presented at national and international scientific conferences to publicise the research to healthcare professionals, health service authorities and the public. A summary of the results will be made available to the study patients if requested.

\section{Patient and public involvement}

Patients and/or the public were not involved in the design, or conduct, or reporting, or dissemination plans of this research.

\section{Author affiliations}

${ }^{1}$ Intensive Care, Franciscus Gasthuis \& Vlietland, Rotterdam, The Netherlands ${ }^{2}$ Department of Intensive Care, Erasmus Medical Centre, Rotterdam, The Netherlands

${ }^{3}$ Department of Internal Medicine, Academic Centre for Thyroid Diseases, Erasmus Medical Centre, Rotterdam, The Netherlands

${ }^{4}$ Pulmonology, Erasmus Medical Centre, Rotterdam, The Netherlands

5 Intensive Care, Ikazia Hospital, Rotterdam, The Netherlands

${ }^{6}$ Intensive Care, Maasstad Hospital, Rotterdam, The Netherlands

Contributors JHV, JvB, E-JW, DG and MEVG conceived the study and initiated the study design. MEVG is the coordinating investigator and grant holder. DG is the principal investigator. TK provided statistical expertise in the clinical trial design, and JHV and TK wrote the statistical analysis plan. JvB, E-JW, JAML and AFCS are the local principal investigators at each study site. All the authors contributed to the refinement of the study protocol and approved the final manuscript. JHV and MPVB wrote the first manuscript draft. JHV and MEH composed the questionnaires used in the study. JHV and MPVB will collect the data and conduct the study.

Funding This study was supported by DSW (for the HORIZON-IC Project; no grant number available), Stichting Theia (grant number: 2020286), Stichting SGS (grant number: 2020355) and BeterKeten (for the HORIZON-IC Project; no grant number available)

Disclaimer The funding sources had no role in the design of the study and collection, analysis, and interpretation of data nor in writing the manuscript.

Competing interests None declared.

Patient consent for publication Not required.

Provenance and peer review Not commissioned; externally peer reviewed.

Supplemental material This content has been supplied by the author(s). It has not been vetted by BMJ Publishing Group Limited (BMJ) and may not have been peer-reviewed. Any opinions or recommendations discussed are solely those of the author(s) and are not endorsed by BMJ. BMJ disclaims all liability and responsibility arising from any reliance placed on the content. Where the content includes any translated material, BMJ does not warrant the accuracy and reliability of the translations (including but not limited to local regulations, clinical guidelines, terminology, drug names and drug dosages), and is not responsible for any error and/or omissions arising from translation and adaptation or otherwise.

Open access This is an open access article distributed in accordance with the Creative Commons Attribution Non Commercial (CC BY-NC 4.0) license, which permits others to distribute, remix, adapt, build upon this work non-commercially, and license their derivative works on different terms, provided the original work is 
properly cited, appropriate credit is given, any changes made indicated, and the use is non-commercial. See: http://creativecommons.org/licenses/by-nc/4.0/.

\section{ORCID iDs}

Johan H Vlake http://orcid.org/0000-0002-0532-0252

Margo MC van Mol http://orcid.org/0000-0002-0213-6054

Michel E van Genderen http://orcid.org/0000-0001-5668-3435

\section{REFERENCES}

1 Azoulay E, Pochard F, Kentish-Barnes N, et al. Risk of post-traumatic stress symptoms in family members of intensive care unit patients. Am J Respir Crit Care Med 2005;171:987-94.

2 Jones C, Skirrow P, Griffiths RD, et al. Post-traumatic stress disorderrelated symptoms in relatives of patients following intensive care. Intensive Care Med 2004;30:456-60.

3 Anderson WG, Arnold RM, Angus DC, et al. Posttraumatic stress and complicated grief in family members of patients in the intensive care unit. J Gen Intern Med 2008;23:1871-6.

4 Gries CJ, Engelberg RA, Kross EK, et al. Predictors of symptoms of posttraumatic stress and depression in family members after patient death in the ICU. Chest 2010;137:280-7.

5 Lautrette A, Darmon M, Megarbane B, et al. A communication strategy and brochure for relatives of patients dying in the ICU. $N$ Engl J Med 2007;356:469-78.

6 Davidson JE, Jones C, Bienvenu OJ. Family response to critical illness: postintensive care syndrome-family. Crit Care Med 2012;40:618-24

7 Anderson WG, Arnold RM, Angus DC, et al. Passive decision-making preference is associated with anxiety and depression in relatives of patients in the intensive care unit. J Crit Care 2009;24:249-54.

8 Siegel MD, Hayes E, Vanderwerker LC, et al. Psychiatric illness in the next of kin of patients who die in the intensive care unit. Crit Care Med 2008;36:1722-8.

9 Shaw RJ, Bernard RS, Deblois T, et al. The relationship between acute stress disorder and posttraumatic stress disorder in the neonatal intensive care unit. Psychosomatics 2009;50:131-7.

10 Lefkowitz DS, Baxt C, Evans JR. Prevalence and correlates of posttraumatic stress and postpartum depression in parents of infants in the neonatal intensive care unit (NICU). J Clin Psychol Med Settings 2010;17:230-7.

11 Miles MS, Holditch-Davis D, Schwartz TA, et al. Depressive symptoms in mothers of prematurely born infants. J Dev Behav Pediatr 2007;28:36-44.

12 Needham DM, Davidson J, Cohen $\mathrm{H}$, et al. Improving long-term outcomes after discharge from intensive care unit: report from a stakeholders' conference. Crit Care Med 2012;40:502-9.

13 Harvey MA, Davidson JE. Postintensive care syndrome: right care, right now... and later. Crit Care Med 2016;44:381-5.

14 van Beusekom I, Bakhshi-Raiez F, de Keizer NF, et al. Reported burden on informal caregivers of ICU survivors: a literature review. Crit Care 2016;20:16.

15 Vermeir J, Holley A, Lipman J. "Not out of the woods"--a wife's perspective: bedside communication. Intensive Care Med 2016;42:446-7.

16 Balluffi A, Kassam-Adams N, Kazak A, et al. Traumatic stress in parents of children admitted to the pediatric intensive care unit. Pediatr Crit Care Med 2004;5:547-53.

17 Black MD, Vigorito MC, Curtis JR, et al. A multifaceted intervention to improve compliance with process measures for ICU clinician communication with ICU patients and families. Crit Care Med 2013;41:2275-83.

18 Shirley ED, Sanders JO. Patient satisfaction: implications and predictors of success. J Bone Joint Surg Am 2013;95:e69-1.

19 Dorman-Ilan S, Hertz-Palmor N, Brand-Gothelf A, et al. Anxiety and depression symptoms in COVID-19 isolated patients and in their relatives. Front Psychiatry 2020;11:581598.

20 Montauk TR, Kuhl EA. COVID-related family separation and trauma in the intensive care unit. Psychol Trauma 2020;12:S96-7.

21 Shafipour V, Moosazadeh M, Jannati Y, et al. The effect of education on the anxiety of a family with a patient in critical care unit: a systematic review and meta-analysis. Electron Physician 2017;9:3918-24.

22 Zante B, Camenisch SA, Schefold JC. Interventions in post-intensive care syndrome-family: a systematic literature review. Crit Care Med 2020;48:e835-40.

23 Bekelis K, Calnan D, Simmons N, et al. Effect of an immersive preoperative virtual reality experience on patient reported outcomes: a randomized controlled trial. Ann Surg 2017;265:1068-73.
24 Koo C-H, Park J-W, Ryu J-H, et al. The effect of virtual reality on preoperative anxiety: a meta-analysis of randomized controlled trials. J Clin Med 2020;9. doi:10.3390/jcm9103151. [Epub ahead of print: 2909 2020].

25 Ryu J-H, Park S-J, Park J-W, et al. Randomized clinical trial of immersive virtual reality tour of the operating theatre in children before anaesthesia. Br J Surg 2017;104:1628-33.

26 Noben L, Goossens SMTA, Truijens SEM, et al. A virtual reality video to improve information provision and reduce anxiety before cesarean delivery: randomized controlled trial. JMIR Ment Health 2019;6:e15872.

27 Maples-Keller JL, Bunnell BE, Kim S-J, et al. The use of virtual reality technology in the treatment of anxiety and other psychiatric disorders. Harv Rev Psychiatry 2017;25:103-13.

28 Mishkind MC, Norr AM, Katz AC, et al. Review of virtual reality treatment in psychiatry: evidence versus current diffusion and use. Curr Psychiatry Rep 2017;19:80.

29 Kothgassner OD, Goreis A, Kafka JX, et al. Virtual reality exposure therapy for posttraumatic stress disorder (PTSD): a meta-analysis. Eur J Psychotraumatol 2019;10:1654782.

30 Wechsler TF, Kumpers F, Muhlberger A. Inferiority or even superiority of virtual reality exposure therapy in phobias? A systematic review and quantitative meta-analysis on randomized controlled trials specifically comparing the efficacy of virtual reality exposure to gold standard in vivo exposure in agoraphobia, specific phobia, and social phobia. Front Psychol 1758;2019:10.

31 Vlake JH, Wils E-J, van Bommel J, et al. Virtual reality tailored to the needs of post-ICU patients: a safety and immersiveness study in healthy volunteers. Crit Care Explor 2021;3:e0388.

32 Vlake JH, Wils E-J, Van Bommel J. Intensive care specific virtual reality (ICU-VR) to improve Post-Intensive care syndrome-related psychological sequelae in survivors of critical illness. ICMx 2020;8.

33 Zigmond AS, Snaith RP. The hospital anxiety and depression scale. Acta Psychiatr Scand 1983;67:361-70.

34 Weiss DS. The impact of event scale: revised. Cross-cultural assessment of psychological trauma and PTSD: Springer, 2007: 219-38.

35 Ware JE, Sherbourne CD. The mos 36-item short-form health survey (SF-36). I. conceptual framework and item selection. Med Care 1992;30:473-83.

36 Aaronson NK, Muller M, Cohen PD, et al. Translation, validation, and norming of the Dutch language version of the SF-36 health survey in community and chronic disease populations. J Clin Epidemiol 1998;51:1055-68.

37 Rensen A, van Mol MM, Menheere I, et al. Quality of care in the intensive care unit from the perspective of patient's relatives: development and psychometric evaluation of the consumer quality index 'R-ICU'. BMC Health Serv Res 2017;17:77.

38 Brück E, Schandl A, Bottai M, et al. The impact of sepsis, delirium, and psychological distress on self-rated cognitive function in ICU survivors-a prospective cohort study. J Intensive Care 2018;6:2

39 Bienvenu OJ, Williams JB, Yang A, et al. Posttraumatic stress disorder in survivors of acute lung injury: evaluating the impact of event scale-revised. Chest 2013;144:24-31.

40 Creamer M, Bell R, Failla S. Psychometric properties of the impact of event scale - revised. Behav Res Ther 2003;41:1489-96.

41 de Miranda S, Pochard F, Chaize M, et al. Postintensive care unit psychological burden in patients with chronic obstructive pulmonary disease and informal caregivers: a multicenter study. Crit Care Med 2011;39:112-8.

42 Bjelland I, Dahl AA, Haug TT, et al. The validity of the hospital anxiety and depression scale. An updated literature review. J Psychosom Res 2002;52:69-77.

43 Sukantarat KT, Williamson RCN, Brett SJ. Psychological assessment of ICU survivors: a comparison between the hospital anxiety and depression scale and the depression, anxiety and stress scale. Anaesthesia 2007;62:239-43.

44 Ware JE, Kosinski M, Keller S. SF-36 physical and mental health summary scales. In: A user's manual 2001, 1994

45 Lacerda M, Ardington C, Leibbrandt M. Sequential regression multiple imputation for incomplete multivariate data using Markov chain Monte Carlo, 2007.

46 Lydersen S. Last observation carried forward. Tidsskr Nor Laegeforen 2019;139. doi:10.4045/tidsskr.19.0061. [Epub ahead of print: 28 May 2019]

47 Benjamini Y, Hochberg Y. Controlling the false discovery rate: a practical and powerful approach to multiple testing. J R Stat Soc 1995;57:289-300.

48 Turon M, Fernandez-Gonzalo S, Jodar M, et al. Feasibility and safety of virtual-reality-based early neurocognitive stimulation in critically ill patients. Ann Intensive Care 2017;7:81. 